\title{
Veber die Zusammensetzung des Nitrobenzids und Sulfobenzids;
}

\author{
ron \\ E. Mitscherlich.
}

1) Das Nitrobenrid.

Reine xiemlich concentrirte Salpetersïure wirkt so wenig auf das Benzin eill, da/s man es damit destilliren lann, ohne dafs es verändert wird. Mit. ranchender Salpetersäure dagegen erwärmt, findet eine Finwirkung unter Wärmeentwickelung statt, weswegen man auch das Benzin nur in lleiner Menge zu der erwürmten Saipetersture hinzusetzen darf; die entstandene Verbindung löst sich in der warmen Salpetersäure vollständig auf, scheidet sich aber beim Frkalten zum Theil wieder daraus $a b$, indem sie, da sie leichter als die Salpetersüure ist, sich auf der Oberfläche der Flüssigheit ansammelt. Verdünnt man die Säure mit Wasser, so sinlt die Verbin. dung zu Boden, da sie schwerer als Wasser ist. Durch Ause. waschen mit Wasser und durch Destilliren kann man sie leicht vollkommen rein erhaltea. Sie bildet eine etwas gelblich geJürbte Flüssigkeit von intensiv süfsem Geschmack und einem eigenthümlichen Geruch, welcher zwischen dem des Bittermandelöls und des Zimmtöls liegt; bei $15^{\circ}$ beträgt ihr specifisches Gewicht 1,209 , sie tocht bei $323^{\circ}$ und destillirt unverändert über. Bei $3^{\circ}$ wird sie fest, indem krystallinische Nadeln die flüssige Masse durchziehen; mit dieser Substanz kann man Salpetersäure destilliren, ohne dafs sie sich rerändert. Mit verdünnter Schwefelsäure erwärmt, destillirt sie, wenn die Temperatur hoch genug ist, unrerändert über; mit cońcentrirter Sch welèisäure erhitzt, wird sie, unter Ent wickelung von schwefliger Säure und unter starker Färbung der Flüssighteit, serlept. Chlor und Brom durch die Flüssigheir 


\section{6}

geleitet, wirken nicht daraul; wird jedoch Chlor mil dena Dampfe der Substanz durch ein heilses Pohr geleitet, so findet eine Zersetzung statt, indem sie Chlorwasserstoffsäure bildet. Mit Kalium erwärmt, detonirt sie so heftig, dals die Gefâfse zersprengt worden; eine wässerige Kaliauflösung wirht nenig darauf ein, Kali in Alkohol aufgelöst zersetzt sie bei der gewöhnlichen Temperatur nicht, damit getocht färbt sich die Auflösung intensiv roth. Westillirt man die rothe Auflösnng, so erhält man eine rothe Substanz, welche bei der gewöhnlichen Temperatur fest ist; ich werde spälerhin darauf zurïckkommen. Ammoniak wirht nicht darauf.

Sie ist in Wasser fast ganz unlöslich, in Alkohol und Aether in jeden Verhältnifs; in concentrirten Säuren, z. B. Salpetersäure und Schwefelsäure, ist sie leicht löslich, bei erhöhter Temperatur mehr als bei niedriger.

0,373 Grm. der Verbindung mit Lupferoxyd rerbrannt, gab 0,57t Hollensäure, worin 0,1593 Koh!e, und 0,2015 Wasser, worin $0,0,26$ Wascerstofl enthalten sind; darnach

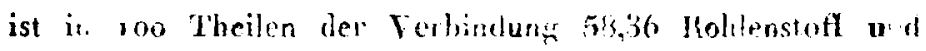
4,123 Wasserstoft enthater.

0,4375 Grm. mit Kupfinoxyd rerbraunt, gaben 1,037 Kohlunsäure, worin 0,287 Kohlenstof, und 0,4775 Wasser, worin 0,0197 Wasserstolf enthalten ist; darnach ist in 100 Theilen der Verbindung 58,70 Kohlenstoff und 4,0/4 Wasserstoff enthalten.

$0,317 \mathrm{Grm}$. der Verbindung gaben $38 \mathrm{C}$. C. Stickstoff bei 760 Nillim. corr. Barometerstand und $15^{\circ}$ Temperatur, darnach enthält die Verbindung 10,6 Proc. Stickstoff. Bei einem zweiten Versnch gaben 0,2505 der Verbindung 25 C. C. Sticlistoffgas von $20^{\circ} 1 / 2$ und 762,6 Millim. corr. Barometerstand; darnach enthält die Verbindung 11,8 Proc Stickstoff. Die Substanz wurde bei diesem Versuch mit Ilupferoxyd nach der gewuhulichen Weiec verbrannt, nachdem ror dom Zer- 
springen der Kugel, welche die Verbindung enthielt, die Luft aus dem Verbrennungsrohr durch Kohlensäure, welche darch Glüben von kohlensanrem Bleioxyd entwickelt wurde, ausgetrieben war; nach dem Verbrennen wurde alles Stickstoffgas, welches noch in dem Rohr zurückgeblieben war, wiederum durch Glïhen einer anderen Menge von lohlensaurem Bleioxyd ausgetrieben. Diese Methode, welche ron Dumas angegeben worden ist, scheint mir fïr diese Bestimmungen ein sehr genaues Resultat za geben.

Nach dem Mittel dieser Versuche besteht daher die Verbindung, da das Fehlende Sauerstoff ist, in 100 Thei!en aus:

$$
\begin{gathered}
58,53 \text { Sauerstoff } \\
4,08 \text { Wasserstoff } \\
11,20 \text { Stickstoff } \\
25,99 \text { Sancrstoff. }
\end{gathered}
$$

Diese Zusanmensetzung liommt folgender Zusaumensetzung so nabe, dals man lieine andere für richtiger aisehen darf, nach der nämlich die Verbindung besteht aus:

$$
\begin{aligned}
58,92 \text { Kohlenstoff } & =12 \text { Maris Hohtenstoffgas } \\
4,008 \text { Wasserstoff } & =10-\text { Witsserstoffgas } \\
21,37 \text { Sticlistof } & =2-\text { Stichstoffgas } \\
25,69 \text { Sauerstoff } & =4-\text { Sauerstoffgas. }
\end{aligned}
$$

Die Bestimmung des specifischen Gewichts der Substanz in gasförmigen Zustand gab folgendes Resultat:

Corrig. Barometerstand beim Zuschmelzen

Corrig. 'Thermometerstand des Metallbades

Das Hohr mit dem Gase gefüllt wog

Das Hohr mit trockner luft von $10^{\circ}$ und

$$
755^{\mathrm{mm}}, 3
$$

Das Rohr mit Wasser von $15^{\circ}$ gefüllt wog $27^{2,0}$ Grm. An Luft von $15^{\circ}$ war zurückgeblieben $\quad$, 3 c. C. Specifisches Gewicht des Gases $=4,40$.

Zwei andere Versuche, bei welchen ein grofser Ueber- 
schuis der Substanz genommen und die zurïchgeblieberce lutt nicht bestimmt wurde, galen 4,35 und 4,38 . Ua das specifische Gewicht stets etwas böher ausfällt, weil die 'lemperatur im Rohr etwas niedriger ist, als dic des Metallbades, so ist

3 Maafs Hoblenstoffigas $=2,5314$

1 Maafs Nitrobenzidgas $=2 \frac{1}{2}-W_{\text {usserstoffgas }}=0,1720$

$$
\begin{aligned}
& 1 / 2-\text { Stichstoffgas }=0,488 \\
& 1-\text { Sauerstofigas }=\frac{1,1026}{4,2940}
\end{aligned}
$$

Da nun ein Maafs Salpetersäure sehr wahrscheinlich aus $1 / 2$ Maafs Stickstoffgas und $1 \%$ Maafs zunerstoffgas besteh so hat sich also ein Maafs Salpetersäurrogas mit einem Maa Benzin zu cinem Maafs Nitrobenzidga:s yerbunden, index 1/2 Maafs Wasserstoff gas und $1 / 4$ Maaís Samerstoffgas aus de Verbindung sich ausgeschieden haben.

2) Das Sulfobenzid.

Setzt man zu Nordhäuser Virrolöl so lange in kleinen Mengen Benzin biszu: lis beim I inschüttein nichis mehr davon anfgelüst wird, so sonder $i$ sich, wenn man die Flïs. sigkeit mit Wassir verdürnt, mit etwas Benzin, welches in Ueberschufs zugesetzt war, eine krystallinische Substanz in so geringer Menge aus, dafs sie haum, bis 2 Procent vom angewandten Benzin beträgt. Mit koblensaurer Baryterde gesättigt und durch scbwefolsaures Kupferoxyd zerlegt, hrystallisiren ans der so crhaltenen Flüssiglueit, wenn man sie vollständig rerdampfen lïist, lírystalle von einer Verbindung von Kupferoxyd mit der Süure des löslichen Barytsalzes her.aus, benzinschwefelsaures Ifupferoxyd nämlich. Setzt man dagegen Benzin zu wasserfreier Schwefelsäure hinzu, so erbält man, ohne dafs in Mindesten eine Zersctoung des Benzins erfolgt, sich folglich keine Spur ron schweflichter Säure 
zeigt, eine zöhe Flüssigkeit, welche in wenig Wasser sich rollkommen auflöst, aus der aber, wenn sie mit viel Wasser rorsetzt wird, sich die krystallinische Substanz in gröfserer Menge aussondert, so da?s man vom angewandten Benzin an 5 bis 6 Proc. von dieser Substanz erbält. Sättigt man die Säure mit Baryt und zersetzt das lösliche Barytsalz mit schwefelsaurem Kupferoxyd, so krystallisirt beim Abdampfen des Kupfersalzes zuweilen nichts, zuweilen nur ein Theil horats, der das gewöhnliche benzinschwefelsaure Iupferoxyd ist; ein anderer ' $T$ 'heil, und zwar die grölsere Menge, scheider sich als unlarstallinisches Pulver beim Eintrochnen der Auflüsung a). Do in.1! sin ähnliches Salz erhält, wenn man die krys..1linische Substanz mit erwärmter concentrirter Schwefelsäu sehandelt, so halte ich es für wahrscheinlich, dal's die aicht trystallisirte Klupfersalz eine Säure enthält, welche sicl arch Einwirkung der Schwefelsäure auf die Imystallinis , Substanz bildet. In einer Notiz, weiche ich bald üler die susammensetzung dieser Säure bekannt machen werle, werde ich auf dieses Salz wieder zurückkommen.

Die hrystallinische Substanz, welche nur sehr wenig in Wasser löslich ist, kann man durch Auswaschen mit Wasser von der anhängenden Säure vollständig reinigen; um sie ganz rein zu erbalten, löst man sie auf in Aether, filtrirt die Auflösung und läfst sie hrystallisiren, die Irystalle destillirt man.

Man kann diese Verbindung, welche in Aether und Al. Hobol löslich ist, durch Verdampfen dieser Auflösungen, in bestimmbaren Krystallen erhalten. Sie schmilzt bei $100^{\circ} \mathbf{z u}$ einer durchsichtigen farblosen Flüssigheit und tocht bei einer Temperatur, welche zwischen dem Hochpuntt des Quecksil. bers und Schwefels liegt; sie ist farblos und geruchlos. In Altalien ist sie unlöslich, in Süuren löst sie sich auf und wird durch Wasser daraus gefällt; mit Schwefelsäure erlhizzt.

Anual. d. Pharn. XII. Bds. 2, u. 3. Heft. 
vorbindet sie sich damit zu einer eigenthümlichen Sänre. "Ach: sich mit der Baryterde zn einem löslichen Salze verbiralet; von den übrigen Säuren wird sie nicht verändert. Wit Salpeter her mit chlorsaurem Kali gemengt uni prhitzt, lit'st sie sich ohne Zersetzen abdestilliren: in starh erhitzten schmelzenden Salpeter, oder in chlorsaures Kali, wetches so stark erhitzt worden ist, dal's es sich zersetzt, geschuittet zerlegt sie sich mit Detonation. Chlor oder Brom wirken bei gewölulicher 'l'emperatur nicht derauf, erhitzt man aber die Substanz bis sie anfängt zu kochen, so wird sie durch Chlor und wuch luych Brom zerlegt, indem sich Chlorbenzin, worsuf ich späticr zurücklommen werde, billet. Nach vielen wigeblichen Vesuchen, hei denen ich diese Substanz über Rupteroxyd, ̈̈lur Gemenge von chlorsaurem Kali mit Chlorlialium oder Kollensaurem Kali leitete, gelang es mir vermittclst dieser Zcisctyung, den Schwefel - und Sauerstoflgehalt, derselben genan zu bestimmen. Den lhohlenstoff- und Wasserstoffgehait bestimmte ich durch Verbre.nen mit Kupferoxyd nach der gewöhnlichen Weise.

0,335 Grm. der Substanz gaben o,801 Grm. Hoblensäure, worin $0,2217 \mathrm{Grm}$. Kohle, und $0,1375 \mathrm{Grm}$. Wasser, worin 0,01555 Wasserstoff enthalien sind.

0,005 Grm. durch Chlor zersetzt, gaben, indern die Prohucts in Ammoniak geleitet wuiden und mil Ammoniak ab. gespïlt worden, 0,304 3chwefetsauren Baryt, worin 0,1045 Sch.refelsiure enibalten sind.

Hundert 'Theile der Substan\% gaben alsn:

$$
\begin{array}{cl}
66,18 & \text { Kohlenstoff } \\
4,552 & \text { Wasserstoff } \\
35,42 & \text { Schwefelsäure } \\
106,152 &
\end{array}
$$

Der Uebersehars bei der Aralyse urd das Tonathits

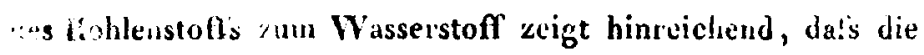


Verbindung auf folgende Weise zusammengeset\%r ist, nänlich aus:

$$
\begin{aligned}
66,42 \text { Kohlenstoff } & =12 \mathrm{C} \\
4,52 \text { Wasserstoff } & =10 \mathrm{H} \\
14,57 \text { Schwefel } & =\mathrm{S} \\
14,49 \text { Sauerstof } & =2 \mathrm{O} .
\end{aligned}
$$

Beide Verbindungen baben sich daher gebilùet, inclem Salpetersüure und Schwefelsäure sich $2 u$ einer neutralen Verbindung mit dem Benzin vereinigt, und indem aus der Verbindung 2 Maals Wasserstoft und I Maals Sauerstoff sich als Wasser ausgeschieden haben. Dieses ist unstreitig der Grund, weswegen diese Verbindung so innig ist, dafs man durch die gewöhnlichen 'Tittel, wodurch man Sïuren auszutreiben pflegt, diese Verbindungen nicht zerlegen kann. Sie sind cin entscheidendes Beispiel dieser Art, und lassen einen directen Schlufs auf die Zusammensetzung einer grofsen Anzahl ron organischen Verbindungen zu. Da diese Substanzen den Amiden am nächsten stehen, so schlage ich für die erstere Jen Namen Nitrobenzid, für die zweite den Namen Sulfobenzid vor. Auf ähnliche Weise kann man die Verbindung, welche man durch Trestillation des festen Chlornaphthaiin, wobei Chlor und Wasserstoff weggehen, Chlornaphthalid nennen.

(Poggend. Annalen Bd. XXXI. S. 625.)

\section{Teber das Stickstoflbenzid;}

\section{von Demselben.}

Wenn Nitrobenzid (= $=12 \mathrm{C}, 0 \mathrm{H} 2 \mathrm{~N} 40)$ mit Kalkerde dcstillirt wird, so wird nur ein höchst unbedeutendi: Theil davon zersetzt; eine wässerige Kaliautlösung wirli gicichlals rur wenig daraur' ein, löst. man das Kali in Altrihol aul und 